\title{
Risk assessment in the first fifteen minutes: a prospective cohort study of a simple physiological scoring system in the emergency department
}

\author{
Tobias M Merz ${ }^{1 *}$, Reto Etter ${ }^{1}$, Ludger Mende ${ }^{1}$, Daniel Barthelmes ${ }^{1}$, Jan Wiegand ${ }^{1}$, Luca Martinolli², Jukka Takala ${ }^{1}$
}

\begin{abstract}
Introduction: The survival of patients admitted to an emergency department is determined by the severity of acute illness and the quality of care provided. The high number and the wide spectrum of severity of illness of admitted patients make an immediate assessment of all patients unrealistic. The aim of this study is to evaluate a scoring system based on readily available physiological parameters immediately after admission to an emergency department (ED) for the purpose of identification of at-risk patients.

Methods: This prospective observational cohort study includes 4,388 consecutive adult patients admitted via the ED of a 960-bed tertiary referral hospital over a period of six months. Occurrence of each of seven potential vital sign abnormalities (threat to airway, abnormal respiratory rate, oxygen saturation, systolic blood pressure, heart rate, low Glasgow Coma Scale and seizures) was collected and added up to generate the vital sign score (VSS). VSS initial was defined as the VSS in the first 15 minutes after admission, VSS $\max$ as the maximum VSS throughout the stay in ED. Occurrence of single vital sign abnormalities in the first 15 minutes and VSS $S_{\text {initial }}$ and VSS $S_{\max }$ were evaluated as potential predictors of hospital mortality.
\end{abstract}

Results: Logistic regression analysis identified all evaluated single vital sign abnormalities except seizures and abnormal respiratory rate to be independent predictors of hospital mortality. Increasing VSS initial and VSS $\max$ were significantly correlated to hospital mortality (odds ratio (OR) 2.80, 95\% confidence interval (CI) 2.50 to $3.14, P<$ 0.0001 for VSS initial; OR 2.36, 95\% Cl 2.15 to $2.60, P<0.0001$ for VSS max). The predictive power of VSS was highest if collected in the first 15 minutes after ED admission (log rank Chi-square 468.1, $P<0.0001$ for VSS initiali, Iog rank Chi square $361.5, P<0.0001$ for $V_{S S_{\text {max }}}$ ).

Conclusions: Vital sign abnormalities and VSS collected in the first minutes after ED admission can identify patients at risk of an unfavourable outcome.

\section{Introduction}

The survival of patients admitted to an emergency department is determined by the severity of acute illness at admission [1] and the level and quality of care provided $[2,3]$. The high number of admissions and the wide spectrum of severity of illness characteristic of large emergency departments make immediate assessment of all patients by an emergency physician unrealistic [4,5]. Various scoring systems have been proposed for identification of patients at risk of deterioration of vital organ functions in the

\footnotetext{
* Correspondence: tobias.merz@insel.ch

'Department of Intensive Care Medicine, Bern University Hospital and

University of Bern, Freiburgstrasse, 3010 Bern, Switzerland

Full list of author information is available at the end of the article
}

emergency department [6-9]. Ideally, the first health care provider encountering the patient should be able to recognize the need for urgent attention within minutes of emergency department admission, without laboratory and radiological examinations or the presence of a specialized physician. Systematic checks for airway, breathing, circulation and level of consciousness are included in resuscitation and trauma guidelines [10,11], and for assessment of risk of deterioration of ward patients in medical emergency team (MET) systems [12-23]. We found in a recent retrospective study that the MET calling criteria were highly predictive of hospital outcome in patients admitted to intensive care from the emergency department [24]. Most emergency departments, including ours, do not

\section{C) Biomed Central}


systematically screen all patients [25]. Even if a scoring system is used, the general concern about the patient's condition, as perceived by the admitting nursing staff, serves as a trigger to expedite evaluation by an emergency physician [26,27].

The time interval until appropriate care is delivered influences outcome in myocardial infarction, stroke, and sepsis [28-32]. It is conceivable that this is also the case for other groups of critically ill patients. One reason for delayed and otherwise suboptimal care is the inability to recognize signs of organ dysfunction early enough to initiate the necessary therapeutic interventions $[13,33,34]$.

The aim of this prospective observational study was to assess the incidence of measurable vital sign abnormalities at admission to the emergency department and the potential impact of these factors on treatment delay and outcome in a large group of unselected patients needing hospital admission. We hypothesised that a scoring system based on the established MET criteria might aid in early recognition of patients at risk of an unfavourable outcome.

\section{Materials and methods Setting}

The study was performed in the Department of Intensive Care Medicine and the Department of Emergency Medicine of the Bern University Hospital, a 960-bed tertiary care referral academic medical centre, in Bern, Switzerland. The emergency department provides initial evaluation and treatment of all adult patients (age $>15$ years).

\section{Patients and study design}

This prospective cohort study includes all patients admitted to our hospital via the emergency department between 11 June 2007, and 11 January 2008. Data were collected prospectively on study data collection forms during the stay in the emergency department and entered in a database created for the purpose of the study. Patients treated on an outpatient basis were not included. In cases where the data were not duplicated to the study record form by the clinical staff, the research staff extracted the data; the data collection sequence and procedure by the clinical staff remained the same. Collected data included patient demographics, time of emergency department admission and discharge, time of first assessment by a physician, and the primary cause of emergency department admission (respiratory, cardiovascular, neurological, trauma, gastrointestinal or other). The time span between admission to the emergency department and discharge was broken down into a series of time periods ( 0 to 15 minutes, 15 minutes to 1 hour (h), 1 to $2 \mathrm{~h}$, 2 to $4 \mathrm{~h}$, followed by two-hour periods up to $24 \mathrm{~h}$ after emergency department admission) during which the presence of vital sign abnormality was investigated. Based on published MET calling criteria [12,23] assessed parameters were respiratory rate, oxygen saturation, systolic blood pressure, heart rate, Glasgow Coma Scale (GCS), presence of a threatened airway and occurrence of seizures (Table 1). The available ED monitoring system provides values for oxygen saturation (pulse oxymetry), systolic blood pressure (sphygmomanometer), heart rate (electrocardiogram), and respiratory rate (constant current impedance pneumography). Presence of a threatened airway was defined as a necessity for intratracheal suctioning, insertion of oro- or nasopharyngeal tubes, intubation, bronchoscopy and occurrence of seizures as repeated or prolonged (>five minutes) seizures. Occurrence of each of the seven potential vital sign abnormalities (VSS criteria) was considered as one VSS point, and the VSS score was defined as the total sum of all VSS points in one time period. The original MET calling criteria contain the criterion "concern", which was not included in the VSS. "Concern" represents a subjective rating rather than a measurable parameter and was shown to have a low frequency and lack of predictive value in one retrospective study in emergency patients [24]. To evaluate associations between VSS scores and predefined outcome variables, the following definitions were used: VSS $_{\text {initial }}$ denotes the VSS score in the first 15 minutes after admission to the emergency department and VSS $_{\max }$ denotes the maximum VSS score throughout the total stay in the emergency department. Hence, $\mathrm{VSS}_{\text {max }}$ represents the highest sum of VSS criteria occurring simultaneously.

\section{Evaluated predictors and outcome measures}

Occurrence of vital sign abnormality at emergency department admission and during emergency department stay as measured by VSS, time delay between emergency department admission, and first assessment

\section{Table 1 Vital Sign Scoring parameters}

\begin{tabular}{|c|c|}
\hline \multicolumn{2}{|l|}{ Airway } \\
\hline $\begin{array}{l}\text { - threatened } \\
\text { airway: }\end{array}$ & $\begin{array}{l}\text { necessity for intratracheal suctioning, insertion } \\
\text { of oro- or nasopharyngeal tubes, intubation, } \\
\text { bronchoscopy }\end{array}$ \\
\hline \multicolumn{2}{|l|}{ Breathing } \\
\hline - respiratory rate: & respiratory rate $<6 /$ minute or $>36 /$ minute \\
\hline $\begin{array}{l}\text { - oxygen } \\
\text { saturation: }\end{array}$ & $\mathrm{SaO}_{2}<90 \%$ despite supplementary oxygen \\
\hline \multicolumn{2}{|l|}{ Circulation } \\
\hline $\begin{array}{l}\text { - systolic blood } \\
\text { pressure: }\end{array}$ & systolic blood pressure $<90 \mathrm{mmHg}$ \\
\hline - heart rate: & heart rate $<40 /$ minute or $>140 /$ minute \\
\hline \multicolumn{2}{|l|}{ Neurology } \\
\hline - GCS: & Glasgow Coma Scale (GCS) score <13 \\
\hline - seizures: & repeated or prolonged ( $>5$ minutes) seizures \\
\hline
\end{tabular}

Vital Sign Scoring parameters were based on medical emergency team calling criteria, as defined by Buist et al. and Cretikos et al. [12,23]. 
by an emergency physician, as well as the length of stay in the emergency department, were evaluated predictors. The primary outcome measure was hospital mortality; this information was extracted from the hospital database. Secondary outcome was the combined endpoint ICU admission or death in ED. The combined endpoint was chosen to account for the fact that death occurring in the ED before discharge to the ICU was proportionately more frequent in patients with high VSS than in patients with low VSS.

Missing data: In cases where data on vital signs were not entered in the study data collection forms, these data were extracted from the ED patient charts or anaesthesia charts. To analyze potential bias between patients with missing data and the rest of the cohort, age, hospital mortality and VSS scores of these patients were compared with patients whose complete data were collected on the study forms.

\section{Ethical approval and patient consent}

The study was approved by the Ethical Committee of the Canton of Bern, and adheres to the tenets of the Declaration of Helsinki. The need for informed consent was waived provided that purely observational data were collected in conjunction with the normal clinical management. Nevertheless, all patients admitted to the Bern University Hospital are routinely informed of their right to specify whether data related to their stay can be used in observational studies; data of patients who declined were not included in the study.

\section{Statistical analysis}

The data were not normally distributed, and are presented as median and interquartile ranges. Comparison of outcome groups defined on the basis of hospital survival/non-survival was performed using the nonparametric Mann-Whitney test or the Chi-square test, as appropriate. Survival in different groups, defined by the primary cause of emergency department admission, was analyzed by applying categorical logistic regression. The predictive value of VSS to hospital mortality was assessed by univariate logistic regression. To assess survival differences throughout the whole score range groups stratified by VSS scores were compared pair-wise using Pearson's Chi square test. Additionally, Kaplan-Meier survival plots were constructed and log rank and Chi-square tests were used to compare survival in groups stratified by VSS $_{\text {initial }}$ and VSS $_{\text {max }}$. Subjects were censored at the time of hospital discharge. Additionally, receiver operating characteristic (ROC) curves were constructed and the area under the curve (AUC) was calculated to assess the capability of $\mathrm{VSS}_{\text {inital }}$ to discriminate survivors from non-survivors. The prognostic significance of an increase of the VSS score during the stay in the emergency department was assessed in a multivariate logistic regression model including VSS $_{\text {initial }}$ and the increase in VSS points $\left(\mathrm{VSS}_{\max }-\mathrm{VSS}_{\text {initial }}\right)$ as predictors and hospital mortality as outcome parameter. Pearson's Chi-square test was used to assess the value of single VSS criteria with regard to hospital mortality. The results of the single Chi-square tests were compared using Cramer's $\mathrm{V}$ (values ranging from 0 to 1 , with $0=$ no association between variables and $1=$ complete association of variables). Forced entry multivariate logistic regression analysis, with all covariates into the regression model in one block, was used to identify independent predictors of mortality. The correlations between VSS initial $_{\text {scores, }}$ the delay until the first assessment of an emergency physician, and length of stay (LOS) in the emergency department and hospital mortality were assessed in univariate and multivariate logistic regression models, as indicated. The correlation between $\mathrm{VSS}_{\text {initial }}$ and the delay until the first assessment of an emergency physician was assessed using linear regression. In all analyses a $P$-value of 0.05 or less was considered statistically significant. Statistical analyses were performed using the software packages SPSS version 13.0 (SPSS, Inc., Chicago, IL, USA) and GraphPad Prism version 4.02 (GraphPad Software, San Diego, CA, USA).

\section{Results}

\section{Patient characteristics}

A total of 4,416 emergency hospital admissions through the emergency department occurred during the study period. Data on 3,104 patients were collected and entered into their study forms during their stay in the ED. In 1,284 patients, data had to be extracted from the ED patient charts. In 28 patients $(0.6 \%)$, study data on vital sign abnormality were not available; these patients were excluded from the analysis. Thus, a total of 4,388 patients with an overall hospital mortality of $7.2 \%$ were studied (Figure 1). Non-survivors were significantly older and had higher VSS $_{\text {initial }}$ and VSS Vax $_{\max }$ scores than surviving patients. The primary cause of emergency department admission was not correlated with hospital mortality. Non-surviving patients had significantly shorter emergency department and hospital length of stay and were assessed with less time delay by an emergency physician (Table 2). Table 3 summarizes the number of patients and hospital mortality per VSS initial $_{\text {and }}$ VSS max $_{\text {ax }}$ scores.

\section{Survival analysis of VSS scoring}

VSS $_{\text {initial }}$ and VSS $_{\max }$ were both predictors of hospital survival odds ratio (OR) $2.80,95 \%$ confidence interval (CI) 2.50 to $3.14, P<0.0001$ for $\mathrm{VSS}_{\text {initial }}$; OR $2.36,95 \%$ CI 2.15 to $2.60, P<0.0001$ for $\mathrm{VSS}_{\max }$ ). The prognostic accuracy of VSS $_{\text {initial }}$ in predicting hospital outcome was 


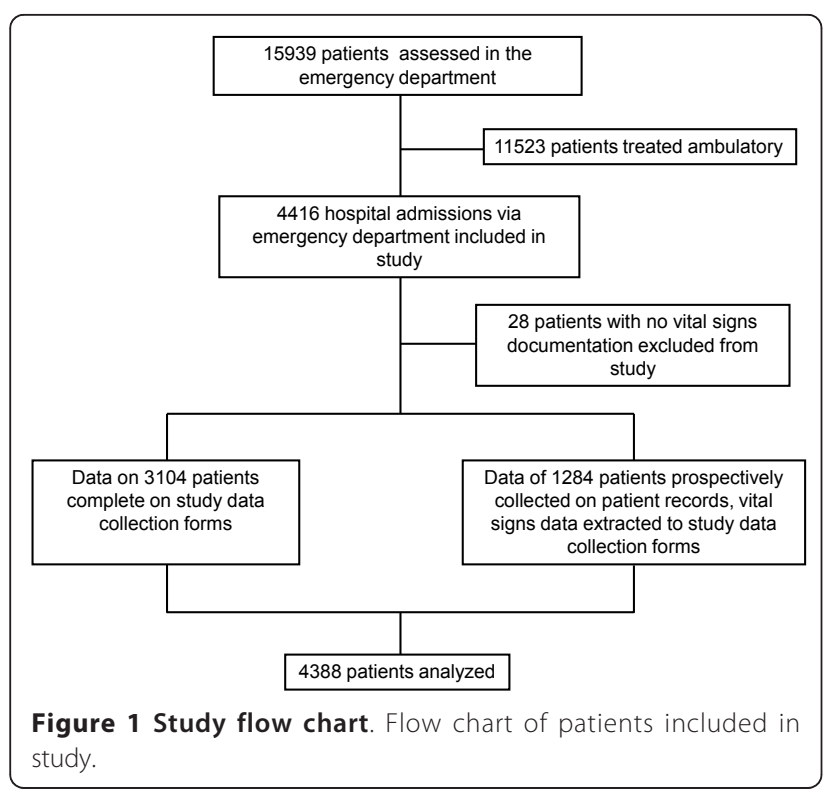

superior to $\mathrm{VSS}_{\max }(\log$ rank Chi-square 468.1, $P<0.0001$ for $V_{S S} S_{\text {initial }}$; log rank Chi square $361.5, P<0.0001$ for

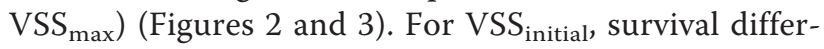
ences were significant over the whole score range except for $\mathrm{VSS}_{\text {initial }} 3$ and 4; for VSS $\mathrm{Vax}_{\text {max }}$ the difference between scores 1 and 2 was not significant (Table 4). Vital sign instabilities developed or increased in 516 patients while in the emergency department $\left(\mathrm{VSS}_{\max }>\mathrm{VSS}_{\text {initial }}\right)$. These patients had a higher mortality than patients in whom the VSS score was highest at admission (OR 1.49, 95\% CI 1.09 to $2.05, P=0.015)$. Figure 4 shows the ROC curve for $\mathrm{VSS}_{\text {initial }}$ plotting sensitivity versus 1 -specificity. The AUC was 0.72 (95\% CI 0.53 to $0.91, P<0.0001)$, indicating a moderately to highly predictive value of $\mathrm{VSS}_{\text {initial }}$ in relation to hospital mortality.

\section{Secondary endpoint ICU admission or death in ED}

$\mathrm{VSS}_{\text {initial }}$ was a significant predictor of the necessity of ICU admission or death in the ED (OR 3.14, 95\% CI 2.80 to $3.52, P<0.0001)$. The secondary endpoint was reached by $14.9 \%$ of patients with a VSS initial of 0 ; respective percentages for $\mathrm{VSS}_{\text {initial }} 1$ to $\geq 4$ were $33.7 \%$, $67.7 \% 75.9 \%$ and $100 \%$.

\section{Prognostic significance of single VSS scoring criteria}

Univariate analysis revealed that all VSS $_{\text {initial }}$ criteria except for seizures were associated with hospital outcome (Table 5). In the multivariate analysis the VSS criteria GCS, systolic blood pressure and oxygen saturation were the most significant independent outcome predictors, followed by heart rate and threatened airway. The criteria respiratory rate and seizures were not independent predictors of hospital mortality (Table 6).

\section{Correlations between scores, delay to first assessment and LOS in the emergency department and hospital mortality}

The delay between emergency department admission and the first assessment by an emergency physician was not a predictor of hospital mortality in a univariate analysis (OR $0.99,95 \%$ CI 0.94 to $1.04, P=0.69$ ) or after correction for vital sign abnormalities at admission $\left(\right.$ VSS $\left._{\text {initial }}\right)$ (OR $0.98,95 \%$ CI 0.94 to $1.04, P=0.65$ ). Shorter LOS in the emergency department was associated with a higher hospital mortality (OR 0.95, 95\% CI 0.92 to $0.98, P<0.0001)$. After correction for vital sign abnormalities at admission $\left(\mathrm{VSS}_{\text {initial }}\right)$, LOS in the

Table 2 Patient characteristics in groups stratified by hospital outcome

\begin{tabular}{|c|c|c|c|c|}
\hline & All patients & Hospital survivors & Hospital non-survivors & $P$-value \\
\hline Number of patients & 4,388 & 4,072 & 316 & \\
\hline Age & $61.0(44.3$ to 74.1$)$ & $60.3(43.0$ to 73.5$)$ & $69.6(57.3$ to 79.7$)$ & $<0.0001$ \\
\hline $\mathrm{VSS}_{\max }$ (points; median/IQR) & $0(0$ to 1$)$ & $0(0$ to 0$)$ & 1 (0 to 2$)$ & $<0.0001$ \\
\hline VSS $_{\text {initial }}$ (points; median/IQR) & $0(0$ to 0$)$ & $0(0$ to 0$)$ & 1 (0 to 2$)$ & $<0.0001$ \\
\hline Primary cause of emergency department admission (\% of patients) & & & & 0.078 \\
\hline Respiratory & $333(7.0)$ & $295(7.2)$ & $38(5.7)$ & \\
\hline Cardiovascular & $633(13.4)$ & $558(13.7)$ & 75 23.7) & \\
\hline Neurological & $895(18.9)$ & $832(20.4)$ & $63(19.9)$ & \\
\hline Trauma & $815(17.2)$ & $776(19.1)$ & $39(12.3)$ & \\
\hline Gastrointestinal & $607(12.8)$ & $570(14.0)$ & $37(11.7)$ & \\
\hline Other & 1,105 (23.3) & $1,041(25.6)$ & $64(20.3)$ & \\
\hline delay first physician (hours; median/IQR) & $0.17(0.0$ to 0.5$)$ & 0.17 (0 to 0.51$)$ & 0.08 (0 to 0.41$)$ & $<0.0001$ \\
\hline length of emergency department stay (hours; median/IQR) & 4.6 (2.8 to 7.3$)$ & 4.6 (2.9 to 7.4$)$ & 4.1 (1.6 to 6.6$)$ & $<0.0001$ \\
\hline length of hospital stay (days; median/IQR) & $6.3(3.0$ to 11.8$)$ & 6.5 (3.1 to 11.8$)$ & $3.4(0.7$ to 11.4$)$ & $<0.0001$ \\
\hline
\end{tabular}

IQR, interquartile range; VSS, Vital Sign Score. 
Table 3 Number of patients and hospital mortality in groups stratified by VSS $_{\text {initial }}$ and VSS max $_{\text {scores }}$

\begin{tabular}{lcccc}
\hline & Number of patients (\%) & Hospital mortality & Number of patients (\%) & VSS \\
& $3,625(82.6 \%)$ & $3.9 \%$ & $3,217(73.3 \%)$ & $3.6 \%$ \\
VSS 0 & $490(11.2 \%)$ & $13.9 \%$ & $577(13.1 \%)$ & $11.6 \%$ \\
VSS 1 & $167(3.8 \%)$ & $25.1 \%$ & $450(10.3 \%)$ & $13.1 \%$ \\
VSS 2 & $58(1.3 \%)$ & $43.1 \%$ & $79(1.8 \%)$ & $36.7 \%$ \\
VSS 3 & $48(1.1 \%)$ & $79.2 \%$ & $65(1.5 \%)$ & $69.2 \%$ \\
VSS $\geq 4$ & & & \\
\hline
\end{tabular}

VSS, Vital Sign Score.

emergency department lost its predictive value for hospital outcome (OR $0.99,95 \%$ CI 0.96 to $1.01, P=0.25$ ).

\section{Missing data}

Patients with complete study form data were slightly younger (median age 59.7 vs $60.8, P=0.009$ ) but had similar hospital mortality $(7.0 \%$ vs. $7.3 \% ; P=0.72)$ as compared to patients whose data were extracted from the patient records. There were no significant differences in the distribution of VSS $\mathrm{Vital}_{\text {ital }}$ groups $\left(\mathrm{VSS}_{\text {inital }} 0\right.$ : $85.0 \%$ vs. $82.5 \%$; $\mathrm{VSS}_{\text {inital }} 1: 7.03$ vs. $12.54 \%, \mathrm{VSS}_{\text {inital }} 2$ : 4.57 vs. $3.31 \%$; $\operatorname{VSS}_{\text {inital }} 3$ : 1.97 vs. $1.11 \%$; $\mathrm{VSS}_{\text {inital }} \geq 4$ : $1.40 \%$ vs $0.48 \% ; P=0.29$ ) between the two groups.

\section{Discussion}

The main finding of this study was that VSS scores based on simple criteria to assess vital sign instability within the first 15 minutes of admission to the emergency department were highly predictive of hospital mortality and necessity of ICU admission in a general population of emergency department patients. The VSS allows for simple and rapid evaluation of patients immediately after emergency department admission by the first health care provider looking after the patient. It may, therefore, facilitate the triage of patients in the emergency department, help caregivers recognize those patients requiring the most urgent attention, and help to avoid delays in implementation of necessary organ

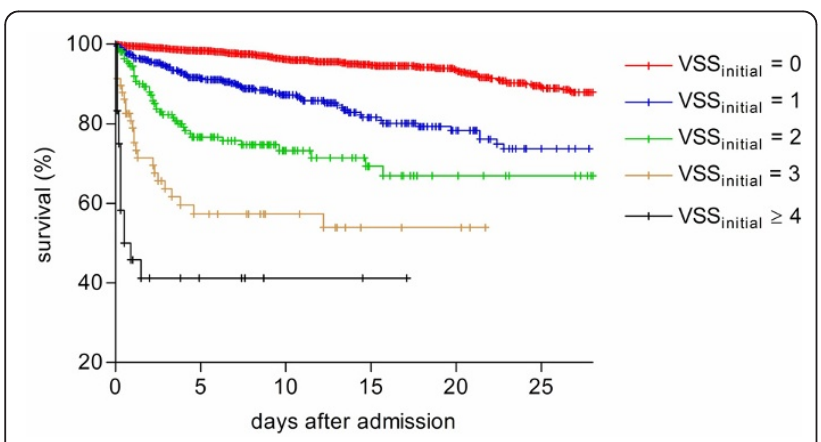

Figure 2 Hospital survival in the strata of VSS $_{\text {initial }}$ groups. Kaplan-Meier plot of hospital survival in the strata of VSS initial groups (log rank Chi-square 468.1, $P<0.0001$ ). function support and commencement of treatment. The sum of single vital sign instabilities is sufficient to obtain the VSS, whereas other reported triage scores $[7,35,36]$ use weighted assessments of vital function parameters and require time-consuming calculations and the use of specific scoring tables. Even if this only takes a few minutes, it might preclude the routine use of these scores in every patient. The prognostic accuracy of the VSS was best if collected early after admission. Whereas VSS $_{\text {initial }}$ represents the patient's condition before the start of treatment, $\mathrm{VSS}_{\max }$ can represent a high score at ED admission and decrease thereafter (positive reaction to resuscitation efforts) or an increase from a lower score (deterioration despite treatment). These two different trends in the patient's condition and reaction to treatment potentially influence the patient's outcome and might explain the difference in the prognostic power of $\mathrm{VSS}_{\text {initial }}$ and $\mathrm{VSS}_{\max }$.

Our results emphasize that the presence, onset, or worsening of vital sign instability in the course of the emergency admission worsens hospital outcome. Not just the initial VSS score but its change during the emergency department stay is relevant: at the same VSS $_{\text {initial }}$ level, patients with increasing VSS scores had higher hospital mortality than those with an unchanged or decreased score in later assessments. We have no data on whether these patients deteriorated despite timely treatment or due to treatment delay.

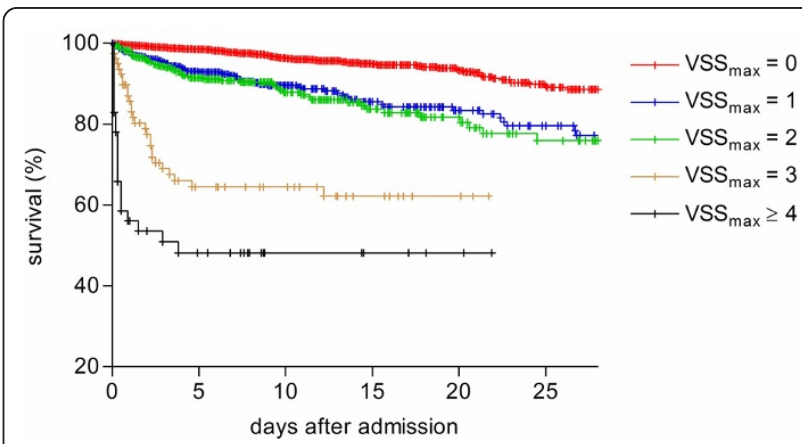

Figure 3 Hospital survival in the strata of VSS max $_{\text {groups. }}$ Kaplan-Meier plot of hospital survival in the strata of VSS $\max$ groups (log rank Chi square 361.5, $P<0.0001$ ). 
Table 4 Survival differences in patient groups stratified by VSS $_{\text {initial }}$ and VSS $_{\max }$ scores

\begin{tabular}{|c|c|c|c|c|c|c|c|c|}
\hline \multicolumn{5}{|c|}{ VSSinitial } & \multicolumn{4}{|c|}{ VSSmax } \\
\hline & Chi-square & OR & $95 \% \mathrm{Cl}$ & $P$ & Chi-square & OR & $95 \% \mathrm{Cl}$ & $P$ \\
\hline VSS 0/1 & 94.31 & 4.10 & 3.03 to 5.54 & $<0.0001$ & 65.7 & 3.45 & 2.54 to 4.77 & $<0.0001$ \\
\hline VSS $1 / 2$ & 11.32 & 2.11 & 1.38 to 3.23 & 0.0008 & 0.89 & 1.22 & 0.84 to 1.76 & 0.35 \\
\hline VSS $2 / 3$ & 13.04 & 3.21 & 1.73 to 5.97 & 0.0003 & 23.23 & 3.63 & 2.14 to 6.17 & $<0.0001$ \\
\hline VSS $3 / 4$ & 0.01 & 1.029 & 0.48 to 2.22 & 0.94 & 8.90 & 2.95 & 1.50 to 5.81 & 0.0029 \\
\hline
\end{tabular}

VSS, Vital Sign Score.

Despite the various physiological triage systems available to identify at-risk patients in the emergency department outcome studies applying these triage scoring systems are scarce and available only in selected subgroups of emergency patients. The concept of adding up the VSS criteria applied in this study is analogous to the use of the sum of failing organs for the calculation of organ dysfunction scores in intensive care [37-39] and we previously used a similar approach for patients admitted to intensive care from the emergency department [24].

It is conceivable that the individual components of the VSS score may have different relevance for the subsequent clinical course. In the present study, impaired levels of consciousness, hypotension, hypoxemia, and abnormal heart rate were the strongest predictors of mortality. In our previous study on patients admitted to intensive care from the emergency department, respiratory rate, decreased level of consciousness, hypoxemia, hypotension, and abnormal heart rate within the first hour in the emergency department were the strongest predictors of mortality. In ward patients, bradypnea, tachypnea, impaired consciousness, high heart rate, low blood pressure, and high respiratory rate were predictors

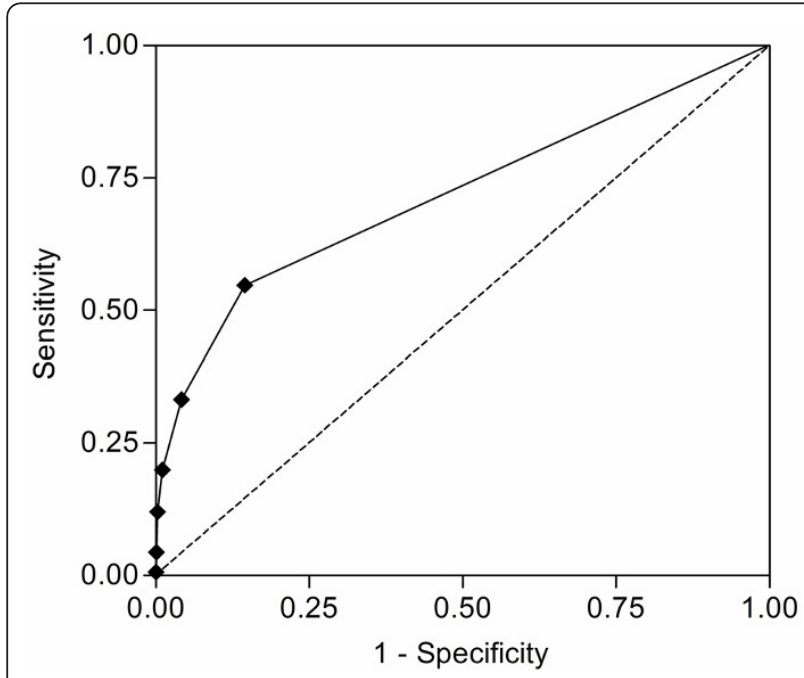

Figure 4 ROC curve for VSS $_{\text {initial. }}$ Receiver operating characteristic

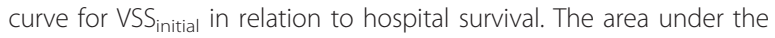
curve was $0.72(95 \% \mathrm{Cl} 0.53$ to $0.91, P<0.0001)$. of mortality [40]. Despite the different patient cohorts and ranking of predictors, all these studies emphasize the relevance of decreased levels of consciousness and cardiovascular and respiratory instability as early predictors of mortality risk.

The lack of independent predictive value for seizures and respiratory rate may be regarded as surprising. Seizures have been associated with increased risk of sudden death [41]. The 56 patients with seizures in this study had a mortality of $8.9 \%$ (vs. $7.8 \%$ for the whole cohort). It is conceivable that the simultaneous presence of other VSS components (for example, hypoxemia and low GCS) may have masked the independent predictive value of seizures. The same can be assumed for respiratory rate: it is likely to have occurred in conjunction with hypoxemia, followed by immediate intubation.

The outcome of critically ill patients in the emergency department can be ameliorated by rapid identification and initiation of appropriate treatment. This is true of ill patients in general [42] and in subgroups such as septic shock [29], trauma [28], acute ischemic stroke [32] and acute myocardial infarction [30]. Optimal management of patients who require advanced organ support seems to be of particular importance, and may have a marked effect on eventual outcome $[43,44]$. The VSS represents a simple scoring system that allows identification of at-risk patients within minutes after arrival. Whether it facilitates rapid commencement of treatment and improves the outcome of these patients is an unanswered question which should be addressed by future research.

The main strength of our study is the use of wellestablished criteria for the evaluation of vital sign abnormalities to generate a simple scoring system, the prognostic value of which was prospectively assessed in patients admitted to the emergency department of a tertiary referral hospital over a period of six months. The analyzed sample size was large and represents a cohort originating from a broad (adult) population covering the whole spectrum of emergencies; all outcomes until hospital discharge were available.

The main limitations of our study are related to the single-centre design and the need to retrospectively extract missing data from patient records. Focusing our 
Table 5 Frequency and results of Chi-square test of single VSS $_{\text {initial }}$ criteria

\begin{tabular}{|c|c|c|c|c|c|c|}
\hline \multirow[t]{2}{*}{ VSS $_{\text {initial }}$ parameter } & \multirow[t]{2}{*}{ Frequency of single VSS criteria (\% of all patients) } & \multirow[t]{2}{*}{ Odds ratio } & \multicolumn{2}{|c|}{$\begin{array}{l}\text { Limits of } 95 \% \text { confidence } \\
\text { interval }\end{array}$} & \multirow[t]{2}{*}{ Cramer's V } & \multirow[t]{2}{*}{$P$-value } \\
\hline & & & lower & upper & & \\
\hline threatened airway & $159(3.6 \%)$ & 9.70 & 6.88 & 13.68 & 0.23 & $<0.0001$ \\
\hline respiratory rate & $80(1.8 \%)$ & 4.84 & 2.90 & 8.08 & 0.10 & $<0.0001$ \\
\hline heart rate & $154(3.5 \%)$ & 5.86 & 3.93 & 8.77 & 0.15 & $<0.0001$ \\
\hline oxygen saturation & $297(6.8 \%)$ & 4.61 & 3.41 & 6.21 & 0.16 & $<0.0001$ \\
\hline systolic blood pressure & $202(4.6 \%)$ & 10.96 & 8.04 & 14. 98 & 0.28 & $<0.0001$ \\
\hline GCS score & $262(6 \%)$ & 12.41 & 9.35 & 16.47 & 0.32 & $<0.0001$ \\
\hline seizures & $56(1.3 \%)$ & 0.0 & 0.0 & 0.0 & 0.01 & 0.99 \\
\hline
\end{tabular}

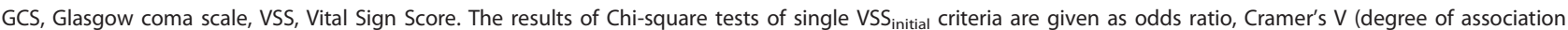
of single VSS criteria and hospital mortality; 0 denoting no association, 1 denoting maximum association) and significance value.

study on hospital admissions and excluding patients treated on an outpatient basis could introduce a selection bias for the study population, as the decision for admission or ambulatory treatment has not yet been made at the time a patient presents at the ED. However, the main outcome parameter of the study was hospital mortality, which can only occur in patients admitted to the hospital. Inclusion of study subjects who by definition cannot reach the main endpoint of the study would confound the results. Whether the VSS score can help to select patients who can be treated as outpatients should be studied separately. Our hospital serves as a primary care centre for a large urban area as well as a tertiary care centre for specialized evaluation and treatment of a population of approximately 1.5 million. With regard to structure and organisation our institution is comparable to other university hospitals in Switzerland and in other countries. Despite the need to extract vital signs data from the patient records in a substantial number of patients, we are confident that this has not biased the main results of the study. All the data needed for the VSS were collected by the same staff as part of their routine clinical work. In cases where the data were not duplicated

Table 6 Results of multivariate logistic regression of individual VSS criteria

\begin{tabular}{lcccc}
\hline VSS & odds ratio & \multicolumn{2}{c}{$\begin{array}{c}\text { limits of 95\% } \\
\text { confidence interval } \\
\text { lower }\end{array}$} & P-value \\
\hline Threatened airway & 1.66 & 1.02 & 2.68 & 0.041 \\
Respiratory rate & 0.74 & 0.36 & 1.54 & 0.42 \\
Heart rate & 2.37 & 1.45 & 3.86 & 0.001 \\
Oxygen saturation & 2.91 & 2.02 & 4.20 & $<0.0001$ \\
Systolic blood pressure & 3.88 & 2.62 & 5.75 & $<0.0001$ \\
GCS score & 6.18 & 4.20 & 9.08 & $<0.0001$ \\
Seizures & 0.83 & 0.31 & 2.26 & 0.83 \\
\hline
\end{tabular}

GCS, Glasgow coma scale; VSS, Vital Sign Score. Results of multivariate logistic regression of individual VSS criteria recorded in the first 15 minutes after emergency department admission, identifying independent predictors given as odds ratio, $95 \%$ confidence interval of odds ratio and significance value for hospital mortality. to the study record form by the clinical staff the research staff extracted the data, the data collection sequence and procedure by the clinical staff were the same. Only in a very small fraction of patients (28 patients) the data for VSS were not available. Furthermore, we found no clinically relevant differences between the characteristics or outcomes in those patients where the vital sign data were collected in both the study form and the patient records vs those with data collected in the patient records only. Finally, since the data were collected without actions to alter the clinical routine, we have no reason to believe that the patients would have been treated differently.

Inter-observer variation in the accuracy of data collection was not assessed. Determination of inter-observer variation of all the involved health care professionals would not have been possible due to the limited study resources. All ED staff had to attend lectures on how to collect the required parameters correctly prior to the study commencement. Parameters were strictly defined and not study specific but part of the already implemented routine clinical data collection. Most data originated from automatic monitoring systems. Therefore, we do not expect a significant bias by high inter-observer variation.

We consider the observed frequency of vital sign instability as a minimum prevalence, since the vital signs were recorded as part of the clinical routine. It is conceivable that the use of continuous monitoring technologies and protocols triggering changes in routine monitoring and treatment based on the observed abnormalities may alter both the detection and occurrence rate of vital sign abnormalities. Finally, only if the detection of vital sign abnormalities triggers the correct intervention can an improvement of outcome be expected. We suggest that the VSS provides a pragmatic approach for structured detection of outcome-relevant vital sign abnormalities and a tool for interventional studies.

\section{Conclusions}

In this prospective cohort study we found that in patients admitted to the emergency department, a score 
derived from readily available physiological parameters registered during the first 15 minutes after admission was strongly associated with the subsequent risk of death. The use of the VSS score in the emergency department may help to design interventions for faster and more systematic identification and treatment of patients at risk of an unfavourable outcome and to avoid delays in implementing organ function support.

\section{Key messages}

- A score (Vital Sign Scoring; VSS) derived from simple criteria to assess vital sign instability within the first 15 minutes of admission to the emergency department is highly predictive of hospital mortality. - The VSS allows for simple and rapid evaluation of patients immediately after emergency department admission by the first health care provider looking after the patient.

- The use of the VSS in the emergency department may help to design interventions for faster and more systematic identification of patients at risk of an unfavorable outcome.

- The VSS may help to avoid delays in treatment and implementation of organ function support in critically ill patients in the emergency department.

\section{Abbreviations}

Cl: confidence interval; ED: emergency department; GCS: Glasgow Coma Scale; LOS: length of stay; MET: medical emergency team; OR: odds ratio; VSS: Vital Sign Scoring

\section{Acknowledgements}

This work was supported by an Innovation Project grant from the Bern University Hospital. Thanks go to the nursing staff and doctors from the Department of Emergency Medicine, Bern University Hospital for their invaluable help with the data collection and to Jeannie Wurz for editorial help.

\section{Author details}

'Department of Intensive Care Medicine, Bern University Hospital and University of Bern, Freiburgstrasse, 3010 Bern, Switzerland. ${ }^{2}$ Department of Emergency Medicine, Bern University Hospital and University of Bern, Freiburgstrasse, 3010 Bern, Switzerland.

\section{Authors' contributions}

$T M, R E, L M e, L M a$ and JT participated in the design of the study. DB designed the study database. RE, DB, LMe and LMa collected all data on ED patients. TM and DB performed the statistical analysis. The manuscript was drafted by TM, assisted by JW and JT. All authors read and revised the manuscript drafts and approved the final manuscript.

\section{Competing interests}

The Department of Intensive Care Medicine has, or has had in the past, research contracts with Abbott Nutrition International, B. Braun Medical AG, CSEM SA, Edwards Lifesciences Services GmbH, Kenta Biotech Ltd, Maquet Critical Care AB, Omnicare Clinical Research AG, and Orion Corporation; and research and development/consulting contracts with Edwards Lifesciences SA, Maquet Critical Care AB, and Nestlé. The money is/was paid into a departmental fund; no author receives/received individual fees. These contracts are unrelated to and did not influence the current study.

Received: 31 May 2010 Revised: 20 December 2010 Accepted: 18 January 2011 Published: 18 January 2011
References

1. Jones $A E$, Fitch MT, Kline JA: Operational performance of validated physiologic scoring systems for predicting in-hospital mortality among critically ill emergency department patients. Crit Care Med 2005, 33:974-978.

2. McQuillan P, Pilkington S, Allan A, Taylor B, Short A, Morgan G, Nielsen M, Barrett D, Smith G, Collins CH: Confidential inquiry into quality of care before admission to intensive care. BMJ 1998, 316:1853-1858.

3. Hillman KM, Bristow PJ, Chey T, Daffurn K, Jacques T, Norman SL, Bishop GF, Simmons G: Antecedents to hospital deaths. Intern Med J 2001, 31:343-348.

4. Derlet RW, Richards JR: Overcrowding in the nation's emergency departments: complex causes and disturbing effects. Ann Emerg Med 2000, 35:63-68.

5. Lynn SG, Kellermann AL: Critical decision making: managing the emergency department in an overcrowded hospital. Ann Emerg Med 1991, 20:287-292.

6. Subbe $C P$, Slater A, Menon D, Gemmell L: Validation of physiological scoring systems in the accident and emergency department. Emerg Med J 2006, 23:841-845.

7. Subbe CP, Kruger M, Rutherford P, Gemmel L: Validation of a modified Early Warning Score in medical admissions. QJM 2001, 94:521-526.

8. Bertollo S, Rodenberg H: Correlation of the RTS (Revised Trauma Score) and RAPS (Rapid Acute Physiology Score) in rotor-wing prehospital care. Air Med J 1994, 13:91-95.

9. Rees JE, Mann C: Use of the patient at risk scores in the emergency department: a preliminary study. Emerg Med J 2004, 21:698-699.

10. 2005 American Heart Association Guidelines for Cardiopulmonary Resuscitation and Emergency Cardiovascular Care. Circulation 2005, 112: IV1-203.

11. Advanced Trauma Life Support ${ }^{\circledR}$ for Doctors $\left(A T L S^{\circledR}\right)$ Student Course Manual. 8 edition. Chicago American College of Surgeons Committee on Trauma; 2002.

12. Buist MD, Moore GE, Bernard SA, Waxman BP, Anderson JN, Nguyen TV: Effects of a medical emergency team on reduction of incidence of and mortality from unexpected cardiac arrests in hospital: preliminary study. BMJ 2002, 324:387-390.

13. Bellomo R, Goldsmith D, Uchino S, Buckmaster J, Hart GK, Opdam H, Silvester W, Doolan L, Gutteridge G: A prospective before-and-after trial of a medical emergency team. Med J Aust 2003, 179:283-287.

14. Lee A, Bishop G, Hillman KM, Daffurn K: The Medical Emergency Team. Anaesth Intensive Care 1995, 23:183-186.

15. Hillman K, Chen J, Cretikos M, Bellomo R, Brown D, Doig G, Finfer S, Flabouris A: Introduction of the medical emergency team (MET) system: a cluster-randomised controlled trial. Lancet 2005, 365:2091-2097.

16. Kenward G, Castle N, Hodgetts T, Shaikh L: Evaluation of a medical emergency team one year after implementation. Resuscitation 2004, 61:257-263.

17. Bellomo R, Goldsmith D, Uchino S, Buckmaster J, Hart G, Opdam H, Silvester W, Doolan L, Gutteridge G: Prospective controlled trial of effect of medical emergency team on postoperative morbidity and mortality rates. Crit Care Med 2004, 32:916-921.

18. Pittard AJ: Out of our reach? Assessing the impact of introducing a critical care outreach service. Anaesthesia 2003, 58:882-885.

19. DeVita MA, Braithwaite RS, Mahidhara R, Stuart S, Foraida M, Simmons RL: Use of medical emergency team responses to reduce hospital cardiopulmonary arrests. Qual Saf Health Care 2004, 13:251-254.

20. Goldhill DR, Worthington L, Mulcahy A, Tarling M, Sumner A: The patientat-risk team: identifying and managing seriously ill ward patients. Anaesthesia 1999, 54:853-860

21. Bristow PJ, Hillman KM, Chey T, Daffurn K, Jacques TC, Norman SL, Bishop GF, Simmons EG: Rates of in-hospital arrests, deaths and intensive care admissions: the effect of a medical emergency team. Med J Aust 2000, 173:236-240

22. Priestley G, Watson W, Rashidian A, Mozley C, Russell D, Wilson J, Cope J, Hart D, Kay D, Cowley K, Pateraki J: Introducing Critical Care Outreach: a ward-randomised trial of phased introduction in a general hospital. Intensive Care Med 2004, 30:1398-1404.

23. Cretikos M, Parr M, Hillman K, Bishop G, Brown D, Daffurn K, Dinh H, Francis N, Heath T, Hill G, Murphy J, Sanchez D, Santiano N, Young L: Guidelines for the uniform reporting of data for Medical Emergency Teams. Resuscitation 2006, 68:11-25. 
24. Etter R, Ludwig R, Lersch F, Takala J, Merz TM: Early prognostic value of the medical emergency team calling criteria in patients admitted to intensive care from the emergency department. Crit Care Med 2008, 36:775-781.

25. Goransson KE, Ehrenberg A, Ehnfors M: Triage in emergency departments: national survey. J Clin Nurs 2005, 14:1067-1074.

26. Patel VL, Gutnik LA, Karlin DR, Pusic M: Calibrating urgency: triage decision-making in a pediatric emergency department. Adv Health $\mathrm{Scl}$ Educ Theory Pract 2008, 13:503-520.

27. Gerdtz MF, Bucknall TK: Triage nurses' clinical decision making. An observational study of urgency assessment. J Adv Nurs 2001, 35:550-561.

28. Nardi G, Riccioni L, Cerchiari E, De Blasio E, Gristina G, Oransky M, Pallotta F, Ajmone-Cat C, Freni C, Trombetta S, Mega AM: [Impact of an integrated treatment approach of the severely injured patients (ISS $=/>16$ ) on hospital mortality and quality of care]. Minerva Anestesiol 2002, 68:25-35.

29. Rivers E, Nguyen B, Havstad S, Ressler J, Muzzin A, Knoblich B, Peterson E, Tomlanovich M: Early goal-directed therapy in the treatment of severe sepsis and septic shock. N Engl J Med 2001, 345:1368-1377.

30. De Luca G, Suryapranata H, Ottervanger JP, Antman EM: Time delay to treatment and mortality in primary angioplasty for acute myocardial infarction: every minute of delay counts. Circulation 2004, 109:1223-1225.

31. Duke G, Green J, Briedis J: Survival of critically ill medical patients is timecritical. Crit Care Resusc 2004, 6:261-267.

32. Tissue plasminogen activator for acute ischemic stroke. The National Institute of Neurological Disorders and Stroke rt-PA Stroke Study Group. N Engl J Med 1995, 333:1581-1587.

33. Goldhill DR, White SA, Sumner A: Physiological values and procedures in the $24 \mathrm{~h}$ before ICU admission from the ward. Anaesthesia 1999, 54:529-534.

34. Hillman KM, Bristow PJ, Chey T, Daffurn K, Jacques T, Norman SL, Bishop GF, Simmons G: Duration of life-threatening antecedents prior to intensive care admission. Intensive Care Med 2002, 28:1629-1634

35. Groarke JD, Gallagher J, Stack J, Aftab A, Dwyer C, McGovern R, Courtney G: Use of an admission early warning score to predict patient morbidity and mortality and treatment success. Emerg Med J 2008, 25:803-806.

36. Rhee KJ, Fisher CJ Jr, Willitis NH: The Rapid Acute Physiology Score. Am J Emerg Med 1987, 5:278-282.

37. Le Gall JR, Lemeshow S, Saulnier F: A new Simplified Acute Physiology Score (SAPS II) based on a European/North American multicenter study. Jama 1993, 270:2957-2963.

38. Knaus WA, Draper EA, Wagner DP, Zimmerman JE: APACHE II: a severity of disease classification system. Crit Care Med 1985, 13:818-829.

39. Vincent JL, Moreno R, Takala J, Willatts S, De Mendonca A, Bruining H, Reinhart CK, Suter PM, Thijs LG: The SOFA (Sepsis-related Organ Failure Assessment) score to describe organ dysfunction/failure. On behalf of the Working Group on Sepsis-Related Problems of the European Society of Intensive Care Medicine. Intensive Care Med 1996, 22:707-710.

40. Buist M, Bernard S, Nguyen TV, Moore G, Anderson J: Association between clinically abnormal observations and subsequent in-hospital mortality: a prospective study. Resuscitation 2004, 62:137-141.

41. Nashef L, Brown S: Epilepsy and sudden death. Lancet 1996, 348:1324-1325.

42. Nguyen HB, Rivers EP, Havstad S, Knoblich B, Ressler JA, Muzzin AM, Tomlanovich MC: Critical care in the emergency department: $A$ physiologic assessment and outcome evaluation. Acad Emerg Med 2000, 7:1354-1361

43. Bion JF, Edlin SA, Ramsay G, MCCabe S, Ledingham IM: Validation of a prognostic score in critically ill patients undergoing transport. $\mathrm{Br}$ Med J (Clin Res Ed) 1985, 291:432-434.

44. Dragsted L, Jorgensen J, Jensen NH, Bonsing E, Jacobsen E, Knaus WA, Qvist J: Interhospital comparisons of patient outcome from intensive care: importance of lead-time bias. Crit Care Med 1989, 17:418-422.

doi:10.1186/cc9972

Cite this article as: Merz et al.: Risk assessment in the first fifteen minutes: a prospective cohort study of a simple physiological scoring system in the emergency department. Critical Care 2011 15:R25. 